\title{
Understanding value-based healthcare - an interview study with project team members at a Swedish university hospital
}

\author{
Annette Erichsen Andersson ${ }^{1,2}$, Fredrik Bååthe ${ }^{1,3}$, Ewa Wikström ${ }^{4}$, Kerstin Nilsson *1 \\ ${ }^{1}$ Institute of Health Care Sciences, Sahlgrenska Academy, University of Gothenburg, Gothenburg, Sweden \\ ${ }^{2}$ The Swedish Institute for Health Science, Lund, Sweden \\ ${ }^{3}$ The Sahlgrenska University Hospital, Gothenburg, Sweden \\ ${ }^{4}$ Department of Business Administration, School of Business, Economics and Law, University of Gothenburg, Gothenburg, \\ Sweden
}

Received: March 12, 2015

DOI: $10.5430 /$ jha.v4n4p64
Accepted: May 3, 2015

Online Published: May 15, 2015

\begin{abstract}
The purpose of this study is to explore how representatives from four project teams understand the concept of value-based healthcare (VBHC), since each representative is responsible for one of the pilot projects implementing VBHC at a university hospital in Sweden. A qualitative design was used to gain understanding of VBHC. Open-ended interviews were used as the data-collection method and content analysis of the transcribed interviews was carried out. Participants' understanding of VBHC focused on how value was created for the patient and on measuring medical outcomes and costs, although costs were to some extent put aside. To measure value for the patients, it was the health professionals' perspective about what patient should value that dominated the understanding of the concept VBHC. VBHC was understood as a strategy to strengthen value innovations and to loosen the grip of economic control. Benchmarking was seen as a future possibility to develop value innovations. Changes in organizational culture were understood by participants as a need to change healthcare from being professional-centred to patient-centred. The way the concept was understood omits parts of the original concept. This has implications for whether or not the concept as it is described by the participants should be understood as VBHC according to the intentions of the strategy described. The development of outcome measures was predominantly based on the health professionals' experiences, which is why the patients' perspective needs to be strengthened. Further studies of the process of implementing VBHC are needed.
\end{abstract}

Key Words: Value-based healthcare, Understanding, Qualitative study, Content analysis

\section{INTRODUCTION}

Value-based management is a growing trend in Swedish healthcare. ${ }^{[1]}$ The implementation of value-based healthcare (VBHC) takes its point of departure in ideas on how to solve problems of quality and economy in healthcare. ${ }^{[2]}$ The founders of this concept are convinced that this demands a shift in focus, i.e. instead of focusing on processes, efforts should be directed towards creating as much value as possible for the patient in relation to cost. ${ }^{[2,3]}$ The overall aim is to develop high quality healthcare, reduce patients' suffering, im-

\footnotetext{
*Correspondence: Kerstin Nilsson; Email: kerstin.nilsson@sahlgrenska.gu.se; Address: Institute of Health Care Sciences, Sahlgrenska Academy, University of Gothenburg, Box 457, 405 30, Göteborg, Sweden.
} 
prove patient safety and achieve better cost-effectiveness. ${ }^{[2]}$ VBHC is expected to stop the rapid escalation of healthcare costs and to lead to better understanding of the total costs per cycle of care for any group of patients. ${ }^{[4]}$ It has been suggested that three principles should guide the implementation of VBHC: first focusing on what creates value for patients, second organizing care praxis around patients' medical conditions and full care cycles, and third measuring medical outcomes and costs. ${ }^{[2,3]}$ This study intends to explore how representatives of four project teams understand the concept of $\mathrm{VBHC}$, since each representative is responsible for one of the pilot projects implementing $\mathrm{VBHC}$ at a large university hospital.

Implementation research has pointed out the importance of understanding the characteristics of the innovation implemented, who is implementing the innovation, the target population for the innovation and the implementation strategies and activities, as well as internal and external organizational factors. ${ }^{[5,6]}$ Over time it was difficult to maintain management innovations as implemented. ${ }^{[7]}$ Results from a crosscase comparative study of the implementation of 12 different organization and management innovations in Swedish healthcare $^{[8]}$ point to the importance of understanding innovations in healthcare as an evolution, where innovations are being adapted and developed over time. They also found that clinical leaders were considered more important than managers for the success of an innovation, that implementation strategies differed according to the type of innovation, and that internal organizational factors were more important than external factors. As has already been mentioned, the implementers of the innovation are of importance for successful implementation. ${ }^{[5,9]}$ Porter and Teisberg ${ }^{[10]}$ emphasize that physician engagement is a key factor when implementing VBHC and that physicians have the authority to change care practice to create value for the patients. However, these authors do not pay any attention to physicians' understanding of what is to be implemented. Lindgren et al. ${ }^{[11]}$ states that if physicians are expected to be engaged in the implementation process they need to understand the innovation in question as a contribution to their professional fulfillment.

Different aspects of VBHC have not been well explored. The evidence for improved health outcomes or reduced healthcare costs as a consequence of VBHC is limited since these approaches are new in healthcare. ${ }^{[12]}$ One of the few studies evaluating the improvement of care in relation to $\mathrm{VBHC}$ has developed and evaluated measurable patient value related to the complete cycles of care of patients suffering from Lynch syndrome, where patients answering a questionnaire emphasized experiencing both adequate patient education and surveillance during their care cycles. ${ }^{[13]}$ A retrospective study involving coordinated implementation of value-based processes across the episode of surgical care of microvascular decompression showed both improved outcome and greater value of the care delivered. ${ }^{[14]}$ Value-based purchasing strategies have also been evaluated by means of peerreviewed and field-based research. ${ }^{[12,15,16]}$ Studies focusing on the understanding of VBHC have not been carried out to the best of our knowledge. Therefore, further studies are needed to understand the characteristics of VBHC better, since understanding what is being implemented is important for successful implementation.

The purpose of this study is to explore how representatives for four project teams understand the concept of VBHC.

\section{METHODS}

\subsection{Design}

A qualitative design ${ }^{[17]}$ was used to explore how the concept VBHC was understood. The research questions were:

- How do the project team members talk about and give meaning to VBHC?

- Which perspectives do the project team members focus on in VBHC?

The data collecting method in this study consisted of openended interviews since the intention was to understand the participants' perspectives and statements. The interviews were carried out during the initial implementation phase of this project, at face-to-face meetings. ${ }^{[18]}$ However, it was the content of the interviews that was in focus in this study, not the interaction in itself. ${ }^{[19]}$

\subsection{Setting}

The study took place at a Swedish university hospital providing highly specialized care and treatment to both children and adults. The hospital had about 2,000 beds distributed between 130 inpatient wards. A total of 16,700 employees provided healthcare for patients during 107,000 care episodes. In addition to inpatient wards the hospital had 180 outpatient wards with 1.2 million visits. In 2013 the hospital management team decided to implement value-based management. The implementation process started with four pilot projects in November 2013. Subsequent planning aims to include all eligible diagnostic groups. The pilot projects focused on bipolar syndrome, prostate cancer, hip arthroplasty and pediatric heart surgery. A project team was established for each pilot project as well as a working team. Patient representatives were invited to participate in each project team on some occasions. The teams were supported during the first three months of the project by consultants from a consulting agency experienced in VBHC. The consultants 
provided methodological experience and knowledge. After establishing the teams and mapping the groups of patients, the plan was to realize the following steps: defining outcome measures and the process of measuring; collecting data; analyzing data; developing and implementing improvement initiatives; and guaranteeing a continuous value-based work method. The continuous feedback from measuring and improving care and treatment are expected to provide organizational learning. The reimbursement system was not changed to the so-called bundle payment system suggested by Porter. ${ }^{[2,3]}$ However, the hospital participated in a national project initiated by The Ministry of Health and Social Affairs concerning bundle payment as well as in a regional project dealing with reimbursement systems.

\subsection{Participants}

Each project team had five members. In each team, one participant had the managerial function of head of department (four persons in all). The functions of the other team members were: healthcare developers (six persons), different health professionals (five persons), controllers (three persons) and logisticians (two persons). Nine of the participants were Senior Consultants (physicians), three were Registered Nurses, three had a Master's Degree in Business Administration, two had a Master of Engineering Degree, two were Psychologists and one was an Occupational Therapist. Thus the teams were not identically composed, but all of them had at least one healthcare developer, at least two physicians and one head of department (also physicians). Three of the project teams had a physician leading the team and one team had a psychologist as team leader. Eleven of the participants were male and nine were female. Their mean age was 47 years old, varying from 37 to 62 years old.

\subsection{Data collection}

Information concerning participants in the pilot project was received from the medical director responsible for the process of implementing VBHC at the hospital. All 20 participants were contacted by telephone and asked if they wanted to participate. All of them agreed. They then received written information on the study and a confirmation of time for the interview. Open-ended questions were based on the aim and the research questions, for example "Can you tell me what VBHC means?" Follow-up questions were asked to clarify and deepen the understanding. The interviews lasted between 37 and 64 minutes (mean 47 minutes). The interviews were carried out at each participant's workplace in a separate room.

\subsection{Data analysis}

The interviews were transcribed verbatim, amounting to about 234 pages of single-spaced text. The transcripts were analyzed as one entity of analysis using content analysis. ${ }^{[17]}$ To gain a holistic sense of the material, all transcripts were read in their entirety by the last-mentioned author. All transcripts were then imported to the programme $\mathrm{NVivo}^{T M} 10$, which was used to facilitate the analysis by organizing units of meaning, nodes, and themes. Keeping the aim of the study in mind, the first step of the analysis was to identify units of meaning and to label them with codes (in Nvivo nodes). Thereafter codes and additional units of meaning were grouped together in terms of content. These groups were reduced or expanded during the continuous process of analysis by identifying differences and similarities. During this process, themes were identified and given headings based on their content. Quotations numbered IP (interview person) 1-20 are used to illustrate the findings.

\subsection{Ethics}

Approval from an Ethical Committee is not needed for interviewing healthcare professionals. ${ }^{[20]}$ The study followed the declaration of Helsinki. ${ }^{[21]}$ The interviewees were informed about the study, confidentiality was assured and informed consent was obtained. All interviewees were given the opportunity to take part in a tentative analysis to confirm that they agreed. All agreed to this analysis except two interviewees who wanted to make minor changes to the wording of two sentences. These wishes were granted.

\section{RESULTS}

Five themes emerged in the analysis describing the participants' understanding of VBHC. These were: 1) The point of departure was seen to be healthcare professionals' view versus the patient's view, 2) The costs perspective, 3) A new method of governance, 4) Benchmarking, and 5) Improvements guided by outcome measures. Participants related their understanding to the value equation (see Equation 1). However they focused mostly on only one of the equation's components. Professional background influenced individual focus.

$$
\text { Value }=\frac{\text { Outcome }}{\text { Costs }}
$$

\subsection{Healthcare professionals' view versus the patient's view as the point of departure}

Participants paid attention to the importance of adopting the patient's perspective. Statements made by healthcare professionals describing VBHC most commonly take the patient as the point of departure. At the same time, it is mainly healthcare professionals who interpret what is of value to the 
patient and they are the ones to identify outcome measures focusing on value for the patient. They stated that the aim of $\mathrm{VBHC}$ is to create more efficient and valuable care for patients. Participants emphasized that creating value for the patients forms the basis of healthcare professionals' ethics and of the nature of health professionals' work. The choice of outcome measures was governed by healthcare professionals' (primarily physicians') assumptions, clinical experience and research concerning what is essential for the patient. Independent of professional background, participants focused on biomedical outcome measures. This focus on biomedical outcome had a tendency to exclude patient values created by care professionals other than physicians. One participant points this out: "Of course it might be valuable to the patient for example to be met kindly or have a soft pillow or receive good hospital food, but VBHC is more about biomedical results and to avoid complications." (IP13).

Significant tools for capturing patients' experiences are the Swedish national quality registers, mainly developed by physicians interested in medical care and treatment and their outcome. However, participants developing outcome measures identified limitations in the quality registers. It takes considerable time to make changes in a national quality register as well as to obtain results. Participants tried to solve this problem in different ways. Even if national quality registers are important not all registered variables are useful as outcome measures when managing healthcare.

While participants discussed healthcare professional-centred care they also talked about the importance of listening to patient representatives and really developing healthcare based on patient reported outcome measures (PROM). If this is actually put into practice then the intentions behind patientcentred care will be realized: "VBHC is all about the patient, seeing the patient as our fellow player, as a partner in the true sense of the word. We have tried to involve patients in the past, for example by letting patient representatives participate in management teams or by allowing patients to tell their stories at meetings; it adds something, but they only represent a few patients. But here (VBHC) we can systematically measure the care we give patients." (IP11).

Participants expressed the idea that VBHC involves asking patients what they expect healthcare to provide for them, instead of healthcare professionals interpreting value outcomes for the patient. Patient representatives participated in all the teams and contributed valuable experiences. Sometimes however their voices were less audible when it came to choosing variables for measurement due to difficulties in introducing new variables into national or other quality registers. Despite these difficulties the focus now is on asking patients about

Published by Sciedu Press how they experience the results of care and treatment. "Actually, now patients evaluate their own care and treatment." (IP12).

\subsection{Denominator costs - a vital but somewhat disre- garded factor}

Participants said that the main point of VBHC is to use the resources to create as much value as possible and to estimate the quality costs for what actually have been done for the patient. Most participants were well aware of the denominator costs and that outcome measures and improvements in healthcare always had to be estimated in relation to their costs. Participants stated that success in implementing $\mathrm{VBHC}$, i.e. in handling costs at the same time as providing valuable healthcare, led success in handling increasing healthcare costs. However, when talking about costs, one participant said that "costs could not just be ignored since costs were supposedly important and an instrument of control. Costs always have to be taken into account." (IP9)

Talking about costs was the controllers' main focus when describing VBHC. They also described the difficulties involved in measuring costs. They said they wanted to measure real patient costs but that was not feasible in today's system. In VBHC it is important to identify and investigate the costs for care provided and relate them to each other in different ways. Participants think that VBHC will contribute to informing taxpayers about the kind of care supplied by the healthcare organization. However this is only of interest if the costs are shown in relation to the quality of healthcare provided: "We are trying to identify the actual costs for care provided and to break down the costs as far as possible, with as little generalization as possible." (IP4).

The fact that VBHC pays attention to costs while at the same time paying attention to outcome measures contributes to healthcare professionals' increased acceptance of registering necessary data. It seems that VBHC appeals to healthcare professionals' closest sphere of interest: "When I discuss costs with physicians and others in the organization, and relate costs to things they are passionate about and responsible for, and then something else happens, they understand." (IP18).

\subsection{Moving from economic control to value innovations}

Participants understood VBHC as a tool to direct and follow up healthcare with a reasonable and clear structure. They talked about the need to develop new measures and a desire to stop using some existing measures that they think are misleading and rough-hewn, such as average length of stay at ward level. Furthermore, they grasped VBHC as a method that documents what has been done. Participants talked about 
VBHC as a tool to create well-founded decisions for developmental work. Outcome measures can provide information about healthcare such as what is good or what is bad, or the dignity of the problem. Based on this knowledge, gathered from participants, steps can be taken to investigate the problem more deeply and more extensively and later on to find solutions. That in turn might lead to developing and establishing new working methods that can subsequently create more value for the patient: "We have to follow up our costs as concretely as possible. We have to monitor how things actually work out for our patients and of course try constantly to improve the healthcare provided. It is part of this concept." (IP4).

Participants stated that one of the most important aspects of VBHC is to manage the total field of undertakings based on outcome measures as evaluated. A goal to strive towards includes more satisfied patients and better results, and to accomplish all this in a less costly way. Health professionals consider that VBHC gives them an opportunity to influence and manage the content of healthcare in favour of the patients. One participant said: "We on the floor have thought we want to do something beneficial to the patient, but have not been able to implement it: 'ouch, ouch, it costs so much'. Now we can do it if the cost is reasonable and if it actually is something that is much better for patient." (IP2).

\subsection{Benchmarking - an activity difficult to define}

Some participants stated that benchmarking belongs to the VBHC concept: "And we should compare ourselves with others who are doing the same thing. If there is someone else who at roughly similar cost has better outcomes, then we can see if they are doing things differently from us, that we could learn from and vice versa." (IP6).

According to the participants, it is essential to develop systems of support in order to implement benchmarking. The national quality register makes it possible to make comparisons with others even if there are often delays. Participants pointed out that if it were to be possible to make and use comparisons, the level of registration of PROM data would have to be improved. The number of patients and respective outcome measures to be registered must be adequate. An inadequate level of registration leads to unreliable results on outcome measures. Some registers have a low level of registered data. According to participants, the use of other outcome measures than those in the national quality register is complicated. A comparison based on these types of data requires the definition of all included variables by the persons making registration for later comparisons. Participants considered that it was a time-consuming task and they questioned its efficiency. However, once these obstacles are things of the past, it would be possible to evaluate healthcare more thoroughly. One participant expressed the problem with benchmarking like this: "The problem with benchmarking that's really important is not to compare apples and pears." (IP10).

Participants stated that if benchmarking is to be a tool to develop healthcare in the future, then they need first to know more about their own measures and results, and about which comparisons are meaningful. Another question involved with whom to make comparisons. Is it necessary to involve more than one other unit? Which would be better, a national or an international context? One participant raised a warning finger concerning benchmarking: "But this isn't just about comparing figures. We have to look at the patient as a whole and all the factors. The more difficult the patient undergoing operation, the worse the results you get." (IP11).

Participants discussed future possibilities for learning through benchmarking and similar types of undertaking in Sweden, while realizing that this requires maturity and the development of a self-critical attitude in the organization. If these requirements are not met, the risk exists that measures detrimental to the participants' own undertakings will be ignored or denied in PROM data. Participants emphasize self-critically that when making comparisons with others, the risk exists of explaining away one's own less satisfactory results by blaming for example case mix, instead of seeing new possibilities of cooperating with and learning from those with whom one is comparing oneself. On the other hand some participants considered it impossible to compare with other organizations even if VBHC strives towards facilitating comparing outcome measures between units and organizations. However comparing how the same unit is developing its outcome measures over time is the pragmatic solution to minimize contextual conflicts.

\subsection{Improvements - the need for changes in organiza- tional culture}

Participants stated that VBHC includes improved working methods and organization of the work. These improvements may be minor or more extensive. Any changes must however be based on the results of outcome measures. Participants noted that making improvements based on the results of outcome measures called for a change in organizational culture since VBHC implied questioning the content of healthcare and how it is provided. At present, implementing VBHC involves putting questions to patients and using unexpected answers as a basis for improvements. Systematic evaluation of the care given according to VBHC may even point to the need to discard current standardized care programmes. For this reason, participants welcomed procedures for measuring 
care and treatment outcomes. Participants considered that VBHC made it possible to identify care and treatment without having any impact on the patient's perceived outcome. One participant explained this as follows: "One group of patients has undergone this or that test or examination, but in fact it makes no difference, if you look at the outcome. We need to strengthen the monitoring of what we are doing. And we may not always do that, when we are very concentrated on the actual treatment of our patients. We think oh, oh, oh, we are carrying out all these investigations, and they show how good we are!" (IP9).

Participants thought that outcome measures implemented within the scope of VBHC might contribute to identifying areas for improvement, but actually making improvements must be done in cooperation with co-workers. Participants emphasized that co-workers' interest in developmental work would probably increase if they could also actually see the changes in outcome measures. If outcome measures were to become an integrated part of work, co-workers could more easily contribute to discussions and solutions concerning areas identified as needing improvement. One participant emphasized the importance of co-workers' participation in developmental work by saying: "It is the healthcare developer's role to follow results and see the trends that go in different directions. But in the change process co-workers must be involved; change management must come from below. It's very important; one can't just run things from the top.” (IP1).

\section{Discussion}

The purpose of this study is to describe how participants understood the concept of VBHC. Their understanding of the concept seemed to be influenced by their professional background. Participants discussed different aspects of VBHC and most of them focused on the importance of creating value for the patients and measuring medical outcomes as well as measuring costs, though to a lesser degree. Participants seemed to understand VBHC as an opportunity to be less governed by economic control. However, there was a certain ambiguity about understanding how to measure value for the patient.

Health professionals took their point of departure in their clinical experiences and in biomedical research-based knowledge when identifying value in care that is possible to measure. However, they stated in contrast that the concept also required them to ask patients how they evaluated the outcome of medical care. Participants without education in healthcare also understood that patients' evaluations were included in the concept but did not offer any suggestion as to how the patients' evaluations should be measured. Instead they re-

Published by Sciedu Press lated value for the patients more generally to costs and care processes.

Participants' focus on outcome measures as a way to improve healthcare for patients is in line with ideas in VBHC. Measuring costs is also in line with these ideas. ${ }^{[2]}$ One of Porter's strategies ${ }^{[22]}$ is to make it mandatory to measure health outcome as he think that this will be a driving force to improve outcomes. Nevertheless, a bone of contention in the continuing implementation of VBHC might be that not all members in the project teams share the same understanding. ${ }^{[23]}$ Accordingly, VBHC appears to be understood as a way to create value for patients with retained or reduced costs. Since the two other components of Porter and Teisberg's $\mathrm{VBHC}^{[2]}$ - creating integrated care units and bundle payment - have more or less been left out here, this raises the question of how VBHC in its present form differs from other management strategies such as Lean, which also focuses on patient value. Lean also emphasizes creating value as defined by the patient and improving quality and efficiency while controlling costs in the provision of optimum patient care. $^{[24,25]}$

Supposing that our aim for healthcare is for it to be ideologically focused on creating value for the patients, it is, according to the participants, in need of cultural change, from health professional-centred to patient-centred care. The question has been raised of the necessity of changing organizational culture in order to improve healthcare, for example in relation to infection control in healthcare. ${ }^{[26]}$ However, the effectiveness of changing organizational culture to improve healthcare performance is questionable. It is difficult to evaluate the effectiveness of strategies to change organizational culture in order to improve healthcare. ${ }^{[27]}$ On the other hand, in a study exploring barriers to lean healthcare implementation, the researchers state that health professionals will commit themselves to change if decisions are kept under their control and if motivation and structure are at the right level. ${ }^{[28]}$ Therefore it is important that health professionals share a common goal when deciding on the desired effects of implementing VBHC.

It is not surprising that individual participants focused on different aspects of VBHC. This can most easily be understood if a social construction perspective is accepted as explanation model. ${ }^{[29]}$ Social reality is a dialectic process between our subjectively and objectively experienced realities. The idea and meaning of VBHC presented by the hospital management team will be interpreted through the participants' subjective experience. Their subjective experiences influence their understanding of what VBHC is and become objectified as reality. This objectified understanding may be understood 
in different ways by individuals in an organization, even though they use the same terminology. ${ }^{[30]}$ However, social interplay between project team members and managers has an impact on the understanding of VBHC. Such interplay calls for communication. Thus, communication in the organization is an important tool for converting socially constructed attitudes and approaches into VBHC. Thus understanding is not automatically given. People learn and make interpretations based on earlier experiences. Their prior understanding of VBHC probably directs the participants' attention and determines what they think is interesting and relevant and what is not. ${ }^{[31]}$

Understanding of VBHC is managed through the definition of significant values for health professionals and is included in the quality registers and values highlighted in these. The health professional perspective of VBHC interacts with quality registers and thus they reinforce each other. The relationship between VBHC and quality registers leads in this way to the dynamic control of values. Furthermore, health professionals themselves become leaders in the enforcement of values because this behaviour is rewarded in quality registers, thereby strengthening health professional-centred care instead of the opposite, patient-centred care which is the main idea of VBHC, i.e. creating value for the patient. ${ }^{[2]}$ The dynamic that comes into being when quality registers created by health professionals are used can seem negative to the patient and to imply a setback although to health professionals the same dynamic might seem positive and to be a reinforcement i.e. of health professionals' control over processes of standardization and also of what is of value to the patient. Even if the quality registers include PROM data such as for example in The Swedish Hip Arthroplasty Register ${ }^{[32]}$ and the National Prostate Cancer Register of Sweden, ${ }^{[33]}$ how can we be certain that these outcome measures signify value for the patients?

The project team members are responsible for the implementation of VBHC within their different areas and as such their roles should not be under-estimated. Their understanding of the concept of VBHC will influence the implementation process. ${ }^{[34,35]}$ The characteristics of the innovation have been identified as important factors for implementation outcome in empirical studies as well as in frameworks for implementation. ${ }^{[9,36-39]}$ References are often made to Rogers' attributes of innovations. ${ }^{[40]}$ Rogers stresses the importance of understanding the characteristics of the innovation, as perceived by the members of a society, as crucial factors to consider when planning an innovation. The degree of adoption can explain the innovation's relative advantage, i.e. it must be perceived as better than the old practice.
That participants in this study appear to adopt VBHC may be explained by the fact that they think that VBHC is a better management model than economic control. Also, compatibility with existing values and past experiences is said to be of importance along with a sound evidence base as a foundation for the innovation. ${ }^{[9,40]}$ At this early stage, the overall impression is that VBHC is seen as being aligned with physicians' professional values. Nevertheless managers need to be aware that physician engagement in improvement work cannot compete with their main interest which is diagnosis and treating patients' disease and illness. This constitutes their core identity as physicians. ${ }^{[41]}$ If the physicians' understanding of $\mathrm{VBHC}$ is not in line with their own professional identity, and seen as contributing to their patients' cure, their engagement in implementing VBHC might be reduced.

Theories about VBHC have been developed from theories of competition and business strategy in business organizations. ${ }^{[42]}$ Would it be possible to manage healthcare by implementing VBHC, combining the participants' understanding with these theories? The findings indicate that health professionals still seem to have a preferential right to interpret what is valuable for the patients. Patient-centred care has been studied for several decades but there is great variation when it comes to the effect on outcome measures. ${ }^{[43]}$ Therefore even more interaction with patients is necessary, as is investigating what patients found of value in relation to their illness and disease. It is clearly essential to know how patients define value, otherwise the risk exists that care development will focus on what is easy to measure instead of what is most important and of greatest value to the patients.

\section{Strengths and limitations of this study}

The main strength of this study was that the participants came from different project teams and had different experiences and perspectives that provided a broad understanding of VBHC. On the other hand, most of the participants were health professionals and that might have contributed to reinforcing the understanding of VBHC as taking its point of departure in professional-centred care. The relatively small sample size and the collection of the data in the initial phase of the implementation process may be seen as a limitation. However, data from the interviews was extensive and rich and gave a very detailed picture of the phenomenon in question, i.e. VBHC. The open-ended interviewing approach contributed to giving the participants opportunities to express their understandings freely. The follow-up questions prevented misunderstandings and made it possible to validate answers during the interviews. The interpretation of the analysis was also a strength due to the fact that the research group had experiences from different research fields and from dif- 
ferent occupational disciplines. Even if data for this study was collected at only one hospital in Sweden, transferring the findings to a wider context would be possible. The findings of this study could well serve as a basis for further studies concerning VBHC.

\section{Conclusions}

The understanding of VBHC focused on creating patient value and measuring bio-medical outcomes and costs; but costs are to some extent de-emphasized in this study. The dominant idea in the understanding of VBHC was that of creating value to the patient. The construction of understanding takes place in relation to the context and other control practices, for example quality registers. The development of outcome measures was predominantly based on the health professionals' perspective. Thus the patients' perspective needs to be strengthened in order to enhance value to patients with regard to outcome measurements.

The way the concept was understood omits several parts of the original concept. VBHC seem not to be completely understood in accordance with the original concept. It has among other things, implications for the value concept as it is described by the participants. Hospital management teams need to be aware of and to manage the implementation of
VBHC based on how differently implementers understand VBHC. Since understanding is based upon a social interplay, management needs to plan for continuous conversations with project team members about the new concept. Management also needs to give enough time for the implementers to reach a common understanding. Thus, interaction and communication in the organization are important tools for transferring socially constructed attitudes. They also make it easier for the implementers to achieve goals in line with the hospital management team's intentions in promoting VBHC.

\section{Authors' contributions}

AEA: Study design, interpretation of the analysis and writing the manuscript; FB: Interpretation of the analysis and writing the manuscript; EW: Interpretation of the analysis and consistency of the manuscript; KN: Study design, data collection, data analysis, interpretation of the analysis and writing the manuscript.

\section{ACKNOWLEDGEMENTS}

We would like to express our gratitude to all the participants.

\section{CONFlicts OF InTEREST Disclosure}

The authors declare that they have no competing interests.

\section{REFERENCES}

[1] Hoglund PJ, Essen A, Choi S, et al. Value-based health care-strategy for efficient Swedish health care. Care should be organized based on what gives value to the patient. Lakartidningen. 2012; 109(47): 2159-61. PMid: 23330480.

[2] Porter ME, Teisberg EO. Redefining Health Care - Creating ValueBased Competition. 2006/06/06 ed. Boston: Harvard Business School Press; 2006 May.

[3] Porter M, Lee TH. The Strategy that will fix health care. Harv Bus Rev. 2013; 91(10): 50-70.

[4] Kaplan RS, Porter ME. How to solve the cost crisis in health care. Harv Bus Rev. 2011; 89(9): 46-52, 4, 6-61 passim. PMid: 21939127.

[5] Wallin L, Ewald U, Wikblad K, et al. Understanding work contextual factors: a short-cut to evidence-based practice? Worldviews Evid Based Nurs. 2006; 3(4): 153-64. PMid: 17177929. http://dx.doi.org/10.1111/j.1741-6787.2006.00067.x

[6] Grol R, Grimshaw J. From best evidence to best practice: effective implementation of change in patients' care. Lancet. 2003; 362(9391): 1225-30. Epub 2003/10/22. PMid: 14568747. http: //dx.doi.org/10.1016/S0140-6736(03) 14546-1

[7] Barnett J, Vasileiou K, Djemil F, et al. Understanding innovators' experiences of barriers and facilitators in implementation and diffusion of healthcare service innovations: a qualitative study. BMC Health Serv Res. 2011; 16(11): 342. http://dx.doi.org/10.1186/147 2-6963-11-342

[8] Ovretveit J, Andreen-Sachs M, Carlsson J, et al. Implementing organisation and management innovations in Swedish healthcare: lessons from a comparison of 12 cases. J Health Organ Manag. 2012; 26(2): 237-57. PMid: 22856178. http://dx.doi.org/10.1108/14777 261211230790

[9] Grol R, Wensing H, Hulscher M, et al. Theories on implementation of change in healthcare. In: Grol R, Wensing H, Hulscher M, Eccles $\mathrm{M}$, editors. Improving patient care: The Implementation of Change in Clinical Practice. Edinburgh: Elsevier; 2005.

[10] Porter ME, Teisberg EO. How physicians can change the future of health care. JAMA. 2007; 297(10): 1103-11. PMid: 17356031 http://dx.doi.org/10.1001/jama.297.10.1103

[11] Lindgren Å, Bååthe F, Dellve L. Why risk professional fulfilment: a grounded theory of physician engagement in healthcare development. Int J Health Plann Manage. 2013; 28(2): e138-e57. http: //dx.doi.org/10.1002/hpm.2142

[12] Eldridge GN, Korda H. Value-based purchasing: the evidence. Am J Manag Care. 2011; 17(8): e310-3. PMid: 21851138.

[13] Hennink SD, Hofland N, Gopie JP, et al. Value-based healthcare in Lynch syndrome. Fam Cancer. 2013; 12(2): 347-54. Epub 2013/05/18. PMid: 23681793. http://dx.doi.org/10.1007/s 10689-013-9655-6

[14] McLaughlin N, Buxey F, Chaw K, et al. Value-based neurosurgery: the example of microvascular decompression surgery. J Neurol Surg. 2014; 120(2): 462-72. http://dx.doi.org/10.3171/2014.5. JNS131996

[15] Tai W, Kalanithi L, Milstein A. What can be achieved by redesigning stroke care for a value-based world? Expert Rev Phar- 
macoecon Outcomes Res. 2014; 14(5): 585-7. PMid: 25095813. http://dx.doi.org/10.1586/14737167.2014.946013

[16] Kavanagh KT, Cimiotti JP, Abusalem S, et al. Moving healthcare quality forward with nursing-sensitive value-based purchasing. J Nurs Scholarsh. 2012; 44(4): 385-95. PMid: 23066956. http://dx.doi.org/10.1111/j.1547-5069.2012.01469.x

[17] Silverman D. Interpreting Qualitative Data Method for Analysing Talk, Text and Interaction. London: Sage Publications; 2001.

[18] Mischler EG. Research interviewing. Context and Narrative. Cambridge: Ma: Harvard University Press; 1986

[19] Edwards D. Discourse and cognition. London, Thousands Oaks, New Delhi: Sage; 1997.

[20] The act. The act of ethical trail of research concerning humans. Stockhom: Ministry of Education and Research; 2003: 460 (Amended SFS 2008: 192).

[21] World Medical Association. World Medical Association Declaration of Helsinki: ethical principles for medical research involving human subjects. JAMA. 2013; 310(20): 2191. http://dx.doi.org/10 1001/jama.2013.281053

[22] Porter ME. A strategy for health care reform-toward a value-based system. The New England Journal of Medicine. 2009; 361(2): 10912. PMid: 19494209. http://dx.doi.org/10.1056/NEJMp0904 131

[23] Aarons GA, Ehrhart MG, Farahnak LR, et al. Aligning leadership across systems and organizations to develop a strategic climate for evidence-based practice implementation. Annu Rev Public Health 2014; 35: 255-74. PMid: 24641560. http://dx.doi.org/10.11 46/annurev-publhealth-032013-182447

[24] Dart RC. Can Lean Thinking transform American health care? Ann Emerg Med. 2011; 57(3): 279-81. Epub 2011/01/14 PMid: 21227539 . http://dx.doi.org/10.1016/j.annemergm ed. 2010.11.027

[25] Toussaint JS, Berry LL. The promise of Lean in health care. Mayo Clin Proc. 2013; 88(1): 74-82. Epub 2013/01/01. PMid: 23274021. http://dx.doi.org/10.1016/j.mayocp. 2012.07.025

[26] Gutacker N, Bojke C, Daidone S, et al. Truly inefficient or providing better quality of care? Analysing the relationship between risk-adjusted hospital costs and patients' health outcomes. Health Econ. 2013; 22(8): 931-47. Epub 2012/09/11. PMid: 22961956. http://dx.doi.org/10.1002/hec. 2871

[27] Parmelli E, Flodgren G, Schaafsma ME, et al. The effectiveness of strategies to change organisational culture to improve healthcare performance. Cochrane Database Syst Rev. 2011; 1: CD008315. Epub 2011/01/21. PMid: 21249706. http://dx.doi.org/10.1002/1 4651858. CD008315.pub2

[28] Hogg FR, Peach G, Price P, et al. Measures of health-related quality of life in diabetes-related foot disease: a systematic review. Diabetologia. 2012; 55(3): 552-65. Epub 2012/01/17. PMid: 22246373. http://dx.doi.org/10.1007/s00125-011-2372-5

[29] Berger P, Luckmann T. The Social Construction of Reality. A Treatise in the Sociology of Knowledge. New York: Penguin Books; 1966.

[30] Sahlin-Andersson K. The meeting between Reform and Practice (In Swedish). In: Zeuthen Bentsen E, editor. When management ambitions meets practice - the challenge of hospital and health services in Denmark and Sweden (In Danish). Copenhagen: Copenhagen Business School Press; 1999. 293-311.
[31] Sandberg J, Targama A. Management and understanding. A Competence Perspectives on Organizations. (In Swedish). Lund: Studentlitteratur; 2013.

[32] Lindgren JV, Wretenberg P, Karrholm J, et al. Patient-reported outcome is influenced by surgical approach in total hip replacement: a study of the Swedish Hip Arthroplasty Register including 42,233 patients. The Bone \& Joint Journal. 2014; 96-B(5): 590-6. Epub 2014/05/03. PMid: 24788491. http://dx.doi.org/10.1302/0 301-620X.96B5. 32341

[33] Tomic K, Sandin F, Wigertz A, et al. Evaluation of data quality in the National Prostate Cancer Register of Sweden. Eur J Cancer. 2015; 51(1): 101-11. Epub 2014/12/04. PMid: 25465187. http://dx.doi.org/10.1016/j.ejca.2014.10.025

[34] Herold DM, Fedor DB, Caldwell S, et al. The effects of transformational and change leadership on employees' commitment to a change: a multilevel study. J Appl Psychol. 2008; 93(2): 346-57. Epub 2008/03/26. PMid: 18361637. http://dx.doi .org/10.10 37/0021-9010.93.2.346

[35] Gifford W, Davies B, Tourangeau A, et al. Developing team leadership to facilitate guideline utilization: planning and evaluating a 3-month intervention strategy. J Nurs Manag. 2011; 19(1): 121-32. Epub 2011/01/13. PMid: 21223412. http://dx.doi .org/10.11 $11 / j .1365-2834.2010 .01140 \cdot x$

[36] Flottorp SA, Oxman AD, Krause J, et al. A checklist for identifying determinants of practice: a systematic review and synthesis of frameworks and taxonomies of factors that prevent or enable improvements in healthcare professional practice. Implement Sci. 2013; 8: 35. Epub 2013/03/26. PMid: 23522377. http://dx.doi.org/10.1186/1 748-5908-8-35

[37] Foy R, MacLennan G, Grimshaw J, et al. Attributes of clinical recommendations that influence change in practice following audit and feedback. J Clin Epidemiol. 2002; 55(7): 717-22. Epub 2002/08/06. http://dx.doi.org/10.1016/S0895-4356(02) 00403-1

[38] Damschroder LJ, Aron DC, Keith RE, et al. Fostering implementation of health services research findings into practice: a consolidated framework for advancing implementation science. Implement Sci. 2009; 4: 50. Epub 2009/08/12. http://dx.doi.org/10.1186/1 748-5908-4-50

[39] Gurses AP, Marsteller JA, Ozok AA, et al. Using an interdisciplinary approach to identify factors that affect clinicians' compliance with evidence-based guidelines. Crit Care. 2010; 38(8 Suppl): S282-91. Epub 2010/08/05. PMid: 20647785. http://dx . doi .org/10.10 97/CCM. Ob013e3181e69e02

[40] Rogers E. Diffusion of Innovations. $5^{\text {th }}$ ed. New York: The Free Press; 2003

[41] Baathe F, Norback LE. Engaging physicians in organisational improvement work. J Health Organ Manag. 2013; 27(4): 479-97. PMid: 24003633. http://dx.doi.org/10.1108/JHOM-02-2012-004 3

[42] Porter ME, Teisberg EO. Redefining competition in health care. Harv Bus Rev. 2004; 82(6): 64-76, 136. PMid: 15202288.

[43] Rathert C, Wyrwich MD, Boren SA. Patient-centered care and outcomes: a systematic review of the literature. Medicare Medicaid Res Rev. 2013; 70(4): 351-79. Epub 2012/11/22. PMid: 23169897. http://dx.doi.org/10.1177/1077558712465774 